Internist 2013 $\cdot 54: 1293-1293$

DOI 10.1007/s00108-012-3228-x

Online publiziert: 17. Oktober 2013

c) Springer-Verlag Berlin Heidelberg 2013

\section{S. Schellong}

Medizinische Klinik 2, Krankenhaus Dresden-Friedrichstadt, Städtisches

Klinikum, Akademisches Lehrkrankenhaus der TU Dresden

\section{Das kranke Bein}

\section{Eine klinische Synopse}

Die Grundkonstellation jeder klinischen Arbeit in Niederlassung und Krankenhaus besteht darin, dass die erste Schilderung des Patienten die Aufmerksamkeit des Arztes zunächst auf ein Organ oder Organsystem lenkt. Von hier aus gilt es, eine möglichst offene Differenzialdiagnose zu beginnen und anschließend durch die vertiefte Anamnese, die körperliche Untersuchung und Zusatzbefunde rasch so einzuengen, dass der weitere Patientenweg Kontur gewinnt. Immer wieder wird daher in Fortbildungen und Übersichten der Weg vom Leitsymptom zur qualifizierten Diagnose gewählt.

Auch in Der Internist sollen in $\mathrm{Zu}$ kunft in loser Folge Schwerpunkthefte diesem Ansatz gewidmet sein. Ausgehend von einer ersten Problemanzeige soll eine breite Palette infrage kommender Diagnosen vorgestellt werden. Dem Leser sollen die wesentlichen klinischen Charakteristika der möglichen Erkrankungen kurz und prägnant so vorgestellt werden, dass deutlich wird, wie weit man mit vertiefter Anamnese und körperlicher Untersuchung zu einer Eingrenzung gelangt. Apparative Zusatzbefunde werden mit dem Stellenwert erläutert, den sie für eine endgültige Klärung besitzen. Therapeutische Erwägungen haben diesem Ansatz folgend weniger Gewicht und werden nur insofern erläutert, als sie frühzeitig bereits bei der Differenzialdiagnose berücksichtigt werden müssen.

Als Auftakt zu dieser Reihe widmet sich das vorliegende Heft einem nur scheinbar in der Peripherie gelegenen Thema der Inneren Medizin, die sich ja primär mit Thorax, Abdomen und Stoffwechsel im weitesten Sinne beschäftigt. Im klinischen Alltag jedoch lenkt der $\mathrm{Pa}$ - tient die Aufmerksamkeit überaus häufig zunächst auf die Beine. Die Beschwerdeschilderung reicht über Schwellungen, Schmerzen und Verfärbungen bis hin zur offenen Wunde. Genau diesen klinischen Erstpräsentationen folgend haben wir die Beiträge in diesem Heft angeordnet. Um eine möglichst enge Absprache zu erreichen und Überschneidungen zu vermeiden, haben wir eine Autorengruppe aus einer einzigen Institution ausgewählt, in diesem Fall aus den Städtischen Kliniken in Dresden.

\section{》) Im klinischen Alltag lenkt der Patient die Aufmerksamkeit überaus häufig auf die Beine}

Der erste Beitrag beschäftigt sich mit der Beinschwellung, die bei einseitigem Auftreten eine lokale Ursache haben muss, bei beidseitigem Auftreten jedoch auf eine allgemeine Ödemkrankheit oder auf eine Fehlfunktion von Herz, Niere oder Leber verweist. Das schmerzhafte Bein wird überwiegend aus neurologischer Perspektive behandelt, in vielen Fällen findet sich jedoch auch eine vaskuläre Ursache. Farbveränderungen an den Beinen haben zumeist eine venöse Ursache, insbesondere aus rheumatologischer und dermatologischer Sicht ergibt sich jedoch eine Vielzahl an Differenzialdiagnosen, deren zunächst verwirrende Vielfalt allein durch die Anamnese und Inspektion geordnet werden kann. Beim offenen Bein dominiert zahlenmäßig wiederum ganz eindeutig die chronische venöse Insuffizienz. Der Beitrag lenkt den Blick aber auf eine größere Zahl alternativer Diagnosen, die in der klinischen Routine oftmals nicht sofort präsent sind. Einen eigenen Beitrag haben wir den Fußläsionen gewidmet. Hier dominiert das diabetische Fußsyndrom mit seinen charakteristischen Läsionen. Auch hier aber gilt es, ein breites Spektrum anderer Erkrankungen zu bedenken.

Entsprechend der Tatsache, dass der körperliche Untersuchungsbefund und damit der klinische Blick der wesentlichste Baustein der Differenzialdiagnose ist, haben wir die Artikel reichlich bebildert. Dem Verlag ist für die gute Ausstattung dieser Ausgabe zu danken. Im Namen aller Autoren hoffe ich, dass das hier gewählte Konzept auf Ihr Interesse stößt und sowohl Anregung als auch Klärung für die klinische Arbeit bedeutet.

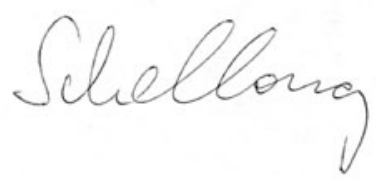

S. Schellong

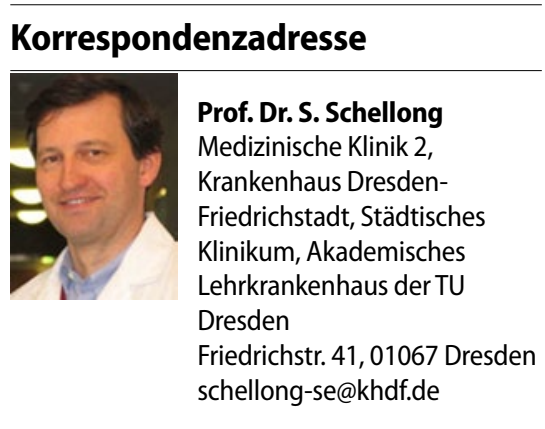

Einhaltung ethischer Richtlinien

Interessenkonflikt. S. Schellong gibt an, dass kein Interessenkonflikt besteht. 